\title{
Students abstract thinking abilities in terms of mathematical disposition
}

\author{
Arfatin Nurrahmah $^{{ }^{*}}$, Lasia Agustina ${ }^{1}$, Nurhayati ${ }^{1}$ \\ ${ }^{1}$ Universitas Indraprasta PGRI, Indonesia \\ $\triangle$ arfatinnurrahmah@gmail.com *
}

Article Information

Submitted April 01, 2021

Revised April 27, 2021

Accepted May 01, 2021

\section{Keywords}

Abstract Thinking Ability; Mathematical disposition.

\begin{abstract}
This research aims to describe students abstract thinking abilities in the probability theory course in terms of mathematical disposition. The research method used is descriptive research with a qualitative approach. The research subjects were 5th-semester students who took probability theory courses. Determination of subjects using purposive sampling techniques. Instruments used include test results of mathematical abstract thinking abilities, disposition questionnaires, interview guidelines, and researchers as the key instruments. Analysis data using the Miles model and Huberman includes data reduction, display data and conclusion drawing/verification. The research results are, Subject 1 (S1) is in the high mathematical disposition category, fulfills three indicators of abstract thinking abilities, namely transforming problems into symbols, manipulation process of abstract mathematical objects, and the formation of mathematical concepts related to other concepts. Subject 2 (S2) is in the medium mathematical disposition category, fulfilling two indicators of abstract thinking abilities, namely transforming problems into symbols and the manipulation process of abstract mathematical objects, the formation of mathematical concepts related to other concepts. Subject 3 (S3) is in the low mathematical disposition category, it only fulfills one indicator of the abstract thinking abilities, namely transforming problems into symbols.
\end{abstract}

\section{INTRODUCTION}

Abstraction is an important construct for mathematics education. Abstraction is a fundamental process in mathematics because abstraction ability allows students to construct mathematical concepts in their minds using initial knowledge (Adelia et al., 2020; Nurhasanah et al., 2017). Abstraction is considered as a high-level knowledge consisting of classifications and generalizations arising from the similarity of certain cases, as well as a construction process, in which the mental framework is designed from a mathematical framework and vice versa (Yilmaz and Argun, 2018). This abstraction is rooted in the deep constructivist view that an individual's initial knowledge is the main source for acquiring new knowledge (Scheiner, 2016a).

Abstraction is considered a highly hierarchical process, where abstraction from a higher level is built on top of a lower abstraction (Scheiner and Pinto, 2016). Hong \& Kim Hong in their research used indicators of mathematical abstraction ability, among others: 1) level I, namely the introduction of mathematical structures through perceptual abstraction; 2) level II, is the application of mathematical structures through internalization; 3) level III, is the development of new mathematical structures through interiorization (Hong and Kim, 2016).

Mathematical abstraction is an essential ability needed to support the process of constructing objects and forming concepts in mathematics (Hakim and Nurlaelah, 2018). The mathematical abstraction ability affects student achievement (Fitriani and Nurfauziah, 2019). 
In addition, mathematical abstraction is often considered as a serious obstacle in mathematics education (Dietz, 2016), especially in advanced mathematics, the process of abstraction will be more difficult because the description and objects are abstract schemas that are formed in mental constructions on previously studied topics (Salgado and Trigueros, 2015). On the other hand, students' abstraction ability is still low and needs to be improved both at the school level (Putra et al., 2018), and at the university level (Widada et al., 2019). (Darwish 2014) Darwish showed that there was a positive correlation between students' abstract thinking test scores and their academic achievement in higher education. It was also reported that there is a gap in the level of abstract thinking, between the current level and the expected level of thinking of students. Students have not reached the minimum abstract level to become a teacher in schools.

The probability theory is a subject that must be studied by students in the study program of mathematics education. Historically, the word chance was associated with the Latin word 'probo' and the English word, probe and probable (Debnath and Basu, 2015). The probability theory and mathematical statistics are complex subjects, because they have systematic and logical characteristics (Wang and Xu, 2018). Students often find it difficult implementing thinking and solving methods in the learning process, many students are less able to understand their knowledge, so it is very important to improve the quality of teaching the probability theory and mathematical statistics. (Wang and $\mathrm{Xu}, 2018$ ).

This probability theory course plays an important role in developing comprehensive abilities and improving students' mathematical achievement. The success or failure of one's learning is not only determined by cognitive abilities, but also by affective abilities (Salido and Dasari, 2019). The affective aspect plays a very important role because it is considered to be able to affect the cognitive aspects. Attitude or disposition aspects in mathematics learning require attention (Lestari et al., 2018). Mathematical disposition can be seen from the way a person learns mathematics, either with confidence, will, perseverance, and interest (Colita and Genuba, 2019). Mathematical disposition affects one's performance when studying mathematics and solving math problems (Sa'Diyah et al., 2019). In this research, the disposition indicators used according to Kusnadi (Hendriana et al., 2017) with modifications include: a) self-confidence; b) flexible; c) persistent, diligent in working on the probability theory task; d) monitoring, reflect on their own appearance and reasoning; e) passionate and serious about learning probability theory; f) expectation and metacognition.

Based on previous researches, many researches have been conducted to analyze students mathematical abstract thinking abilities (Darwish, 2014; Putra et al.,2018; Nurhasanah et al., 2018; Komala, 2018) and research on mathematical dispositions (Colita and Genuba, 2019; Cheng and Wan, 2017; Sa'Diyah et al., 2019; Lin and ChunTai, 2016; Yaniawati et al., 2019; Almerino, Jr. et al., 2019). However, there is no research that describes students abstract thinking abilities in solving the probability theory questions as seen from their mathematical disposition. The novelty of this research is to review the thinking process of students' mathematical abstraction from an affective perspective, namely mathematical disposition. So, in this research, the students' abstract ability to solve the probability theory questions was analyzed as seen from their mathematical disposition. 


\section{METHODS}

This research was conducted at the University of Indraprasta PGRI Jakarta. The method used in this research is descriptive research with a qualitative approach. The research subjects were fifth semester students who take the probability theory course. Determination of the subject using purposive sampling technique. The instruments used include the test document on the results of mathematical abstract thinking abilities, disposition questionnaires, interview guides, and researchers as key instruments (Sugiyono, 2016). The questionnaire was used to collect data related to student attitudes towards mathematics, with a Likert scale answer, namely Strongly Agree (SS), Agree (S), Disagree (TS) and Strongly Disagree (STS). The test is given to analyze students' abilities in solving probability theory questions based on indicators of mathematical abstract thinking abilities. Then, semistructured interviews were conducted with selected subjects based on high, medium and low disposition criteria. The research flow chart is presented in the following figure.

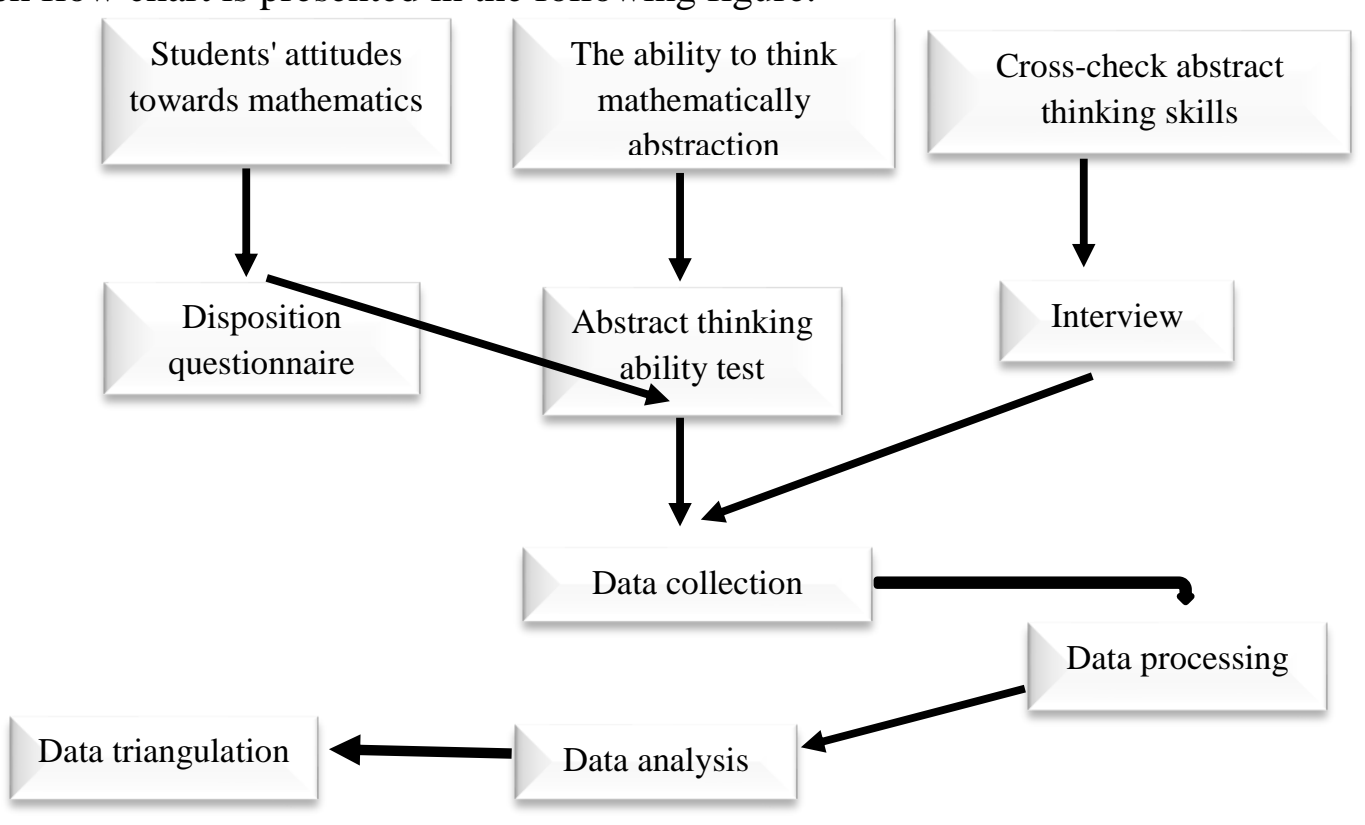

Figure 1. Research Flow Chart

Based on Figure 1, the research flow is detailed as follows, the first thing to do is to distribute a mathematical disposition questionnaire to determine the subject into categories (high, medium, and low dispositions). Second, the implementation of research by providing questions about mathematical abstract abilities regarding the probability theory. The third is analyzing the qualitative data. During the research, analysis was carried out during data collection and after completing data collection within a certain period. Activities in data analysis using the Miles and Huberman model including data reduction, data display, and conclusion drawing/verification. In reduction, researchers summarize and categorize them to make it easier to collect data. Then the data is presented in a narrative form. After that, it can be concluded about students' abstract thinking abilities based on mathematical disposition. Testing the validity of research data using source triangulation.

\section{RESULTS AND DISCUSSION}

The first step taken in this research was to distribute a questionnaire to divide students into several groups based on the level of mathematical disposition in the probability theory course, 
into high, medium and low dispositions. Based on the results of the disposition questionnaire, it is obtained as in the following table.

Table 2. Category of students' mathematical disposition

\begin{tabular}{llll}
\hline Category & High & Medium & Low \\
\hline Number of Students & 7 & 26 & 2 \\
\hline
\end{tabular}

Based on Table 2, using benchmarks to determine the high, medium and low groups using the Mean and Standard Deviation, the number of students who entered the high disposition category was 7 students or $20 \%$, the medium disposition category was 26 students or $74.3 \%$, and low category as many as 2 students or $5.7 \%$. This means that in the probability theory course, students are more dominant in the medium disposition category than the high and low ones. After knowing the mathematical disposition category of students, then the data of mathematical abstract thinking abilities was taken by giving a test on the material of probability theory, namely conditional probabilities and Bayes' argument; the probability function, expectation, and variance of a discrete random variable; as well as the density function, expectation, and variance of continuous random variable. The question consists of four questions and represents each indicator of the ability to think mathematical abstraction, namely: (1) Transforming problems into symbols, (2) Manipulation process of abstract mathematical objects, (3) The formation of mathematical concepts related to other concepts, (4) Construct a general form related to the mathematical process. Data is provided in Table 3 below.

Table 3. Mathematical Abstract Thinking Abilities Data Based on Disposition

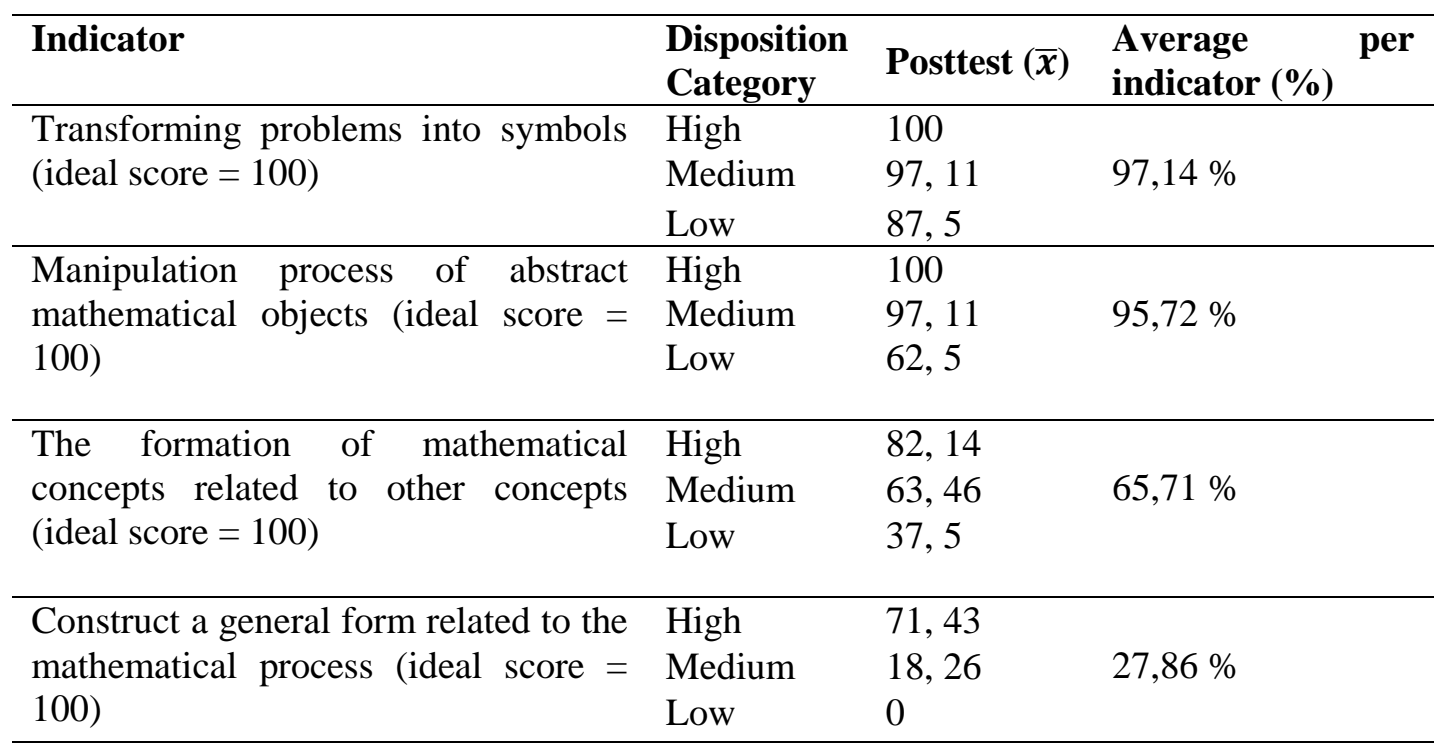

Based on Table 3, it shows that for all indicators of students mathematical abstract thinking abilities in the probability theory course, based on the average achievement per indicator and based on the disposition category, the indicator of "transforming problems into symbols" for the probability function and specific distribution of discrete random variables materials is 97.14\%; the indicator of "manipulation process of abstract mathematical objects" for Bayes's argument is $95.72 \%$; the indicator of "the formation of mathematical concepts related to other 
concepts" for the material of the probability function, expectation, and variance of discrete random variables is $65.71 \%$; and the indicator of "Construct a general form related to the mathematical process" for continuous random variable material is $27.86 \%$.

To find out more about students' abilities in solving mathematical abstract thinking problems in the probability theory course in terms of mathematical disposition, the following will describe further students abstract thinking abilities in solving the probability theory problems. The analysis will be carried out per indicator by taking one student each from each disposition category, hereinafter referred to as S1 (high disposition category students), S2 (medium disposition category students), and S3 (low disposition category students). The following are the results of the analysis based on the abstracti thinking ability indicator and the mathematical disposition category in the oprobability theory course.

\section{Indicator of "Transforming problems into symbols"}

A coin is made in such a way that the head occurs twice as often as the tail. Then the coin is tossed over and over until the head occurs for the first time on the 10th toss. How does the probability function of that event!

The question above aims to reveal the abstract thinking ability on indicator of transforming problems into symbols with the problems given regarding the probability function of an unbalanced currency toss. Figure 2 is the answers from S1, S2, and S3.

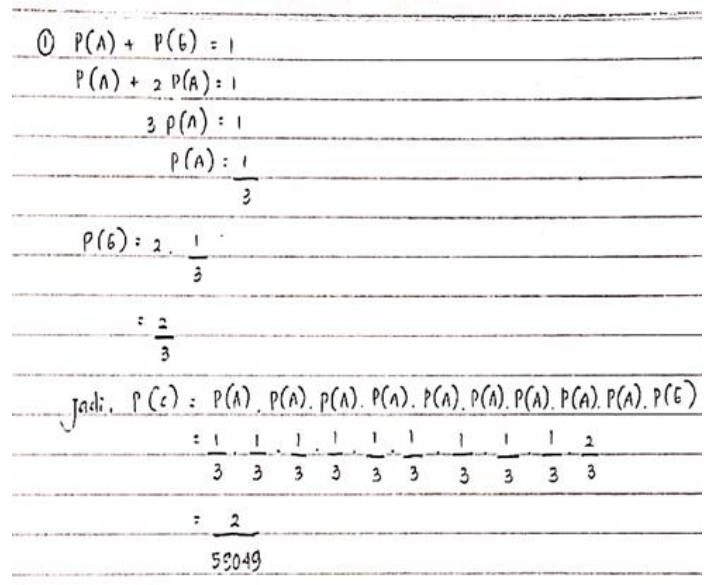

(a)

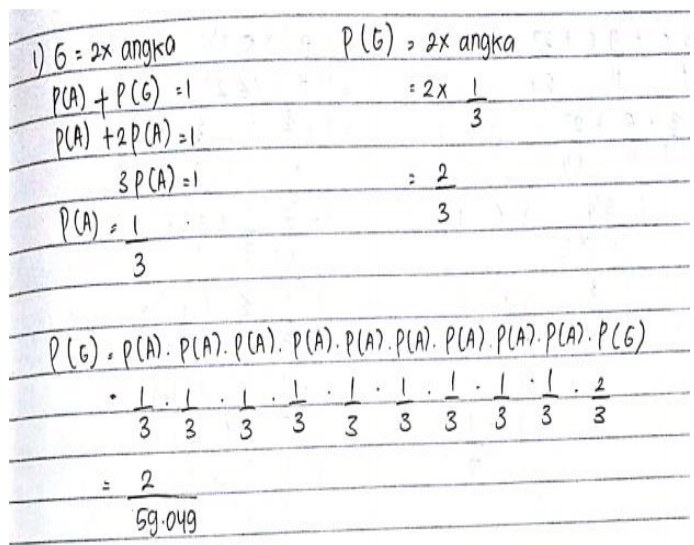

(b)

Figure 2. The answers of S1, S2, and S3 on indicator 1

Based on Figure 2, S1, S2, and S3 can answer this question. S1 can determine each of the probability values of the occurrence of tail and head appearing, then S1 can describe the probability of the occurrence of the head event for the first time on the 10th toss. Here is the result of an interview with one of the subjects (S1) on indicator 1.

P: "For question number 1, how difficult do you think it is? Easy, moderate, or hard?"

S1: "Moderate ma'am, at first I was confused about which formula to use, finally I found it, in the beginning I looked for other references ma'am, and also trying to browse"

$P$ : "Try to explain again how to get the answer"

S1: "Previous because occurrences head $(G)$, two times more often than the occurence of tail (A), then the probabilities are respectively, $P(G)=\frac{2}{3}$ and $P(A)=\frac{1}{3}$. Then, the next question is doing 10 attempts, what is the probability that the head will appear the first time in the tenth 
trial, so when throwing 1 to 9 the tail appears, so I just multiplied the tail probability value by 9 times, then multiplied by the tail probability value."

$P$ "Apart from multiplying the probability values above, do you think there are other events to determine the probability function in question number 1?"

S1: '(thought for a moment).. "yea maybe.. ma'am, but i forgot"

$P$ : "You could actually use a discrete special distribution method, do you remember?"

S1: “ ... (thinking).. "Oh.. yes ma'am, is it geometric discrete special distribution huh?"

Based on the results of the interview, S1 considered that question number 1 had a moderate level of difficulty, because at the beginning of reading the questions S1 was a little confused. However, to answer this question, S1 looked for various references both from sources in handbooks and other sources. This means that S1 has persistence and perseverance as well as passionate and serious concern in learning the probability theory.

Likewise, S2 and S3 which are in the medium and low categories, they said that the questions were at moderate level of difficulty, and like S1, they could answer the questions given well. The following are the results of the interview with S3 on indicator 1. During the interview, S2 and S3 can recall the steps in obtaining the requested probability function, even though S3 forgot a little how to describe the probability value of each event of tail and head. So, based on the analysis results from the test results, interview results, and observations of the three subjects, it can be said that the difficulty level on the indicator of "Transforming problems into symbols" is at moderate level of difficulty, and the three subjects can answer well.

\section{Indicator of "Manipulation process of abstract mathematical objects"}

During November - December 2020, the police conducted Patuh Jaya Operation Raids at 4 different locations in East Jakarta, including on St. Dewi Sartika, St. Jatinegara Barat, St. Otista, St. Pramuka. Each location does not conduct raids all the time but 40\% at St. Dewi Sartika, 30\% at St. Jatinegara Barat, 20\% at St. Otista, and 10\% at St. Pramuka. If Lala wants to go to campus and does not turn on the lights, the probability of getting a ticket is 0.2 at St. Dewi Sartika, 0.1 at St. Jatinegara Barat, 0.5 at St. Otista and 0.2 at St. Pramuka. Determine the probability of Lala will get a ticket at St. Jatinegara Barat!

Question number 2 aims to reveal the abstract thinking process on the indicators of the manipulation process of abstract mathematical objects with the problems given regarding the Bayes argument. Bayes' Theorem is a formula for calculating conditional probability to provide a way to re-examine the flow of expectations (Kontou and Stylianides, 2020). The following are the answers given by S2 and S3. 


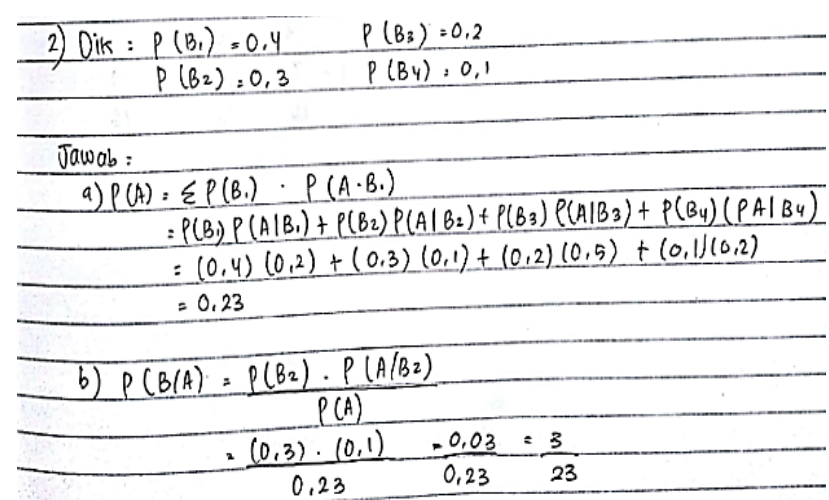

(a)

$$
\begin{aligned}
& \text { 2. a. Peluang lata fertena tilang } \\
& A=J 1 \text { Dewi sartika } \quad P=0,2 \\
& B=J l \text {. Jatinegara barat } P=0,1 \\
& C=J 1 \text { Otista } \quad P=0,5 \\
& D=11 \text { pramular } \quad P=0,2 \\
& \text { poluang lak kena tilang } \\
& P(\text { ii })=(A+B+C+D) \\
& p(m)=(0,2+0,1+0,5+0,2) \\
& p(m)=0,10
\end{aligned}
$$

Figure 3. The answers of $\mathrm{S} 2$ and $\mathrm{S} 3$ on indicator 2

Based on Figure 3 (a), S2, which is in the medium disposition category, can describe the steps in getting a solution for question number 2, by writing down the known elements; write down the total probability proposition generally and determine the value of Bayes' Theorem appropriately. The following are the results of an interview with one of the subjects 2 (S2) on indicator 2 .

P: "For question number 2, how difficult do you think it is? Easy, moderate, or hard?"

S2: "Moderate ma'am, when I read the question, I already knew that it used the Bayes Theorem method, and I have often tried questions like this"

$P$ : "Try to explain again how to get the answer!"

S2: "I initially followed the method by using the general formula for total probabilities in the textbook page 76 ma'am, then following the example in the book, by entering the probability that were already in the question into the general formula for total probabilities while for the question of probabilities that Lala will get a ticket at St. Jatinegara Barat using the Bayes Rule”.

Based on the results of the interview, $\mathrm{S} 2$ considers that question number 2 has a moderate level of difficulty, because when reading the question, $\mathrm{S} 2$ already knew that the solution uses Bayes's proposition, and S2 is used to working on questions like this because S2 prefers to work on questions like the example provided. Based on the results of the disposition questionnaire, S2 felt less enthusiastic about solving problems related to everyday problems. This indicates that S2 is less flexible. Similar to S2, S1 can also do question number 2 correctly. S1 said that he prefers to solve questions related to everyday problems and story questions because he feels more challenged. However, it is different with S3 which is in the low disposition category, unable to solve the question number 2 appropriately. S3 can write down the known elements; however, it was wrong in determining the total probability theorem value generally, as shown in Figure 3 (b). The following are the results of interviews with one of subjects 3 (S3) on indicator 2.

P: "For question number 2, how difficult do you think it is? Easy, moderate, or hard?"

S3: "It's hard ma'am, when I look at question number 2, I seem to have studied, but I didn't find it, maybe I didn't learn it enough, it was my fault that I study less so I doubt my answer ma'am"

Based on the results of the interview, S3 considered that question number 2 was a bit hard. Even though he knows that in solving this question using Bayes's theorem, however, S3 is still wrong in distinguishing the partition probability value and the conditional probability 
value. This causes S3's answer incorrect to the question number 2. S3 prefers to work on the questions as the example and hesitates with their answer. This shows that S3 lacks of confidence and flexibility. So, based on the analysis results of the test results, interview results, and observations of the three subjects, it can be said that the difficulty level on the indicator of "Manipulation process of abstract mathematical objects" is moderate, and the subjects can answer well, except for S3.

Indicator of "The formation of mathematical concepts related to other concepts"

A probability function of: $p(x)=c\left(\frac{3}{4}\right)^{x} ; x=1,2,3 \ldots$.

\section{Determine: $\operatorname{Var} X$}

$$
=0 \quad ; x \text { other }
$$

The question number 3 aims to reveal indicators of the formation of mathematical concepts related to other concepts, where the question given are regarding the expectations and variance of one discrete random variable which has an infinite value of $x$ but can be calculated. Before getting the variance, you must first find the value of $c$ (constant) by using the concept of the probability function. Then, to get the average expected value or $E(X)$ and $E\left(X^{2}\right)$, then they must use the concept of an infinite geometric series, which has been previously studied at senior high school. This means that to solve these questions, students must master several other concepts.

Based on the answers of the subjects, only S1 can answer question number 3, S2 can only answer until they get the value of $c$ (constant), but do not answer until they get the variance value. Meanwhile, S3 did not answer at all.

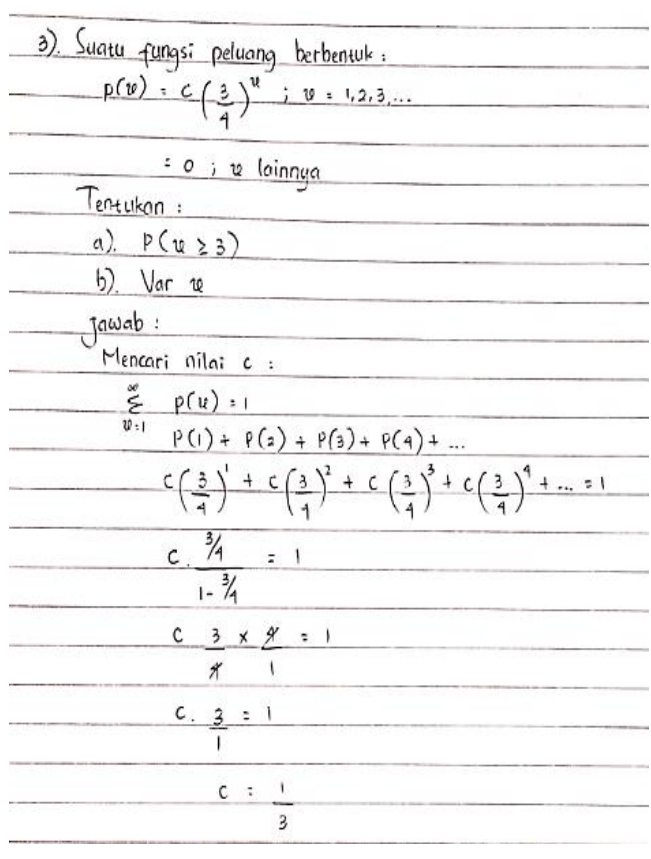

Figure 4. $S_{1}$ answers on indicator 3

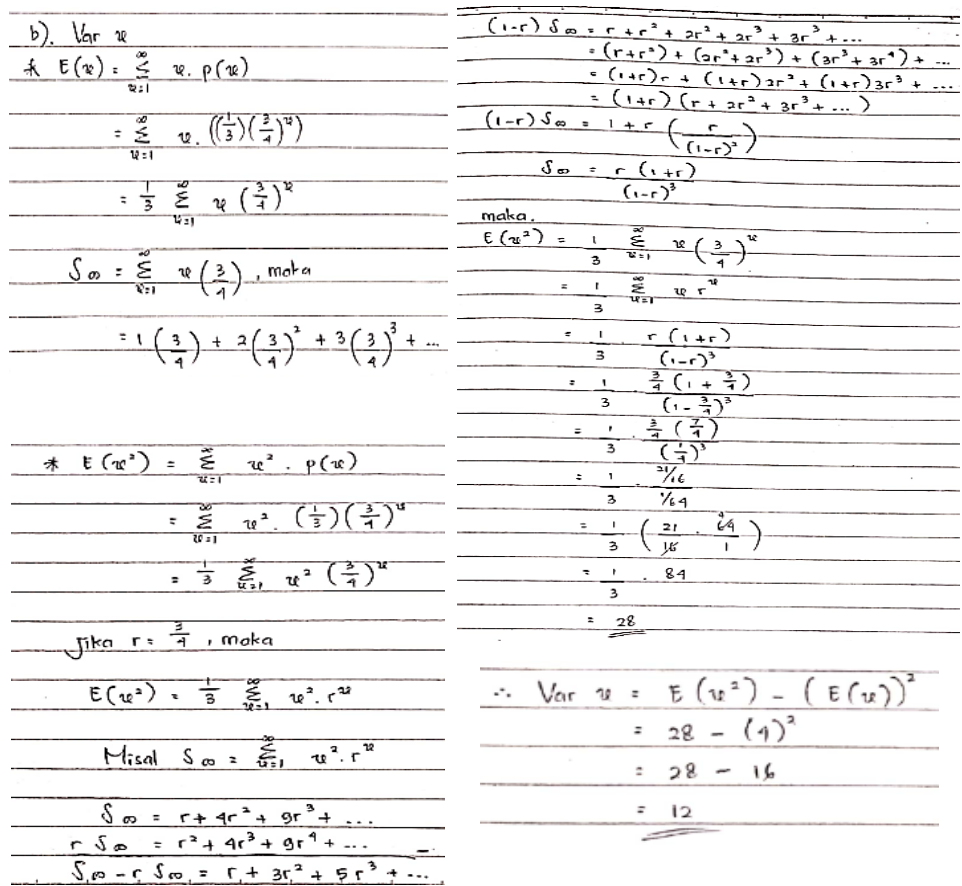

As shown in Figure 4, it can be seen that $\mathrm{S} 1$ can write down the constant value, determine the expected value and the variance of the discrete random variable correctly. Based on the results of the interview, $\mathrm{S} 1$ considers that question number 3 is a little hard, because it requires coherent steps to solve it before getting the variance value and the value of the infinite discrete random variable. However, S1 felt curious when he could not solve the problem 
which he thought was hard. It is different with S1, S2 can find and write constant values, but cannot determine the expected value and the variance of discrete random variables. The following are the results of interviews with one of subjects 2 (S2) on indicator 3.

P: "For question number 3, how difficult do you think it is? Easy, moderate, or hard?"

S2: "It is hard, ma'am"

$P$ : "What makes it hard?".

S2: "Having trouble on determining the right formula, I didn't do it because I didn't understand the infinite value ma'am .."

Based on the results of the interview, S2 did not continue to answer because they did not understand about infinite or infinite values. S2 said that he prefers simple questions to solve, and according to him, the question number 3 is hard. While S3 did not answer the question number 3, even when asked the meaning of $c$ in the question number 3, S3 could not answer. The following are the results of interviews with one of subjects 3 (S3) on indicator 3.

P: "For question number 3, why didn't you answer it?

S3: "I'm already confused because my time is limited ma'am. and in the textbook, I also didn't find example like that so I didn't understand what method to answer it"

When interviewed, S3 said that he had never tried a question like this before and only chose simple questions as an exercise to solve. It shows that S2 and S3 are not persistent and diligent in solving probability theory problems. So, based on the analysis results of the test results, interview results, and observations of the three subjects, it can be said that the difficulty level on the indicator of "The formation of new concepts related to other concepts" is hard, and only the subjects of S1 who can answer, while S2 and S3 cannot answer.

\section{Indicator of "Construct a general form related to the mathematical process (generalization)"}

Given the density function as follows:

$$
\begin{aligned}
f(x) & =\frac{3}{8} x^{2} ; 0<x<2 \\
& =0 ; x \text { other }
\end{aligned}
$$

The density function above has the following data: $E(X)=\frac{3}{2} ; E\left(X^{2}\right)=\frac{12}{5}$

Based on the data above, determine $E\left(X^{n}\right)$. Explain your answer along with the reasons!

The questions given aim to reveal indicators that compose general forms related to the mathematical process (generalization), where the problem given is regarding the density function and the expected value of a continuous random variable, which is then asked to find the general form of $\mathrm{E}\left(\mathrm{X}^{\mathrm{n}}\right)$. The following are the answers from S1 and S2 to the question number 4 . 


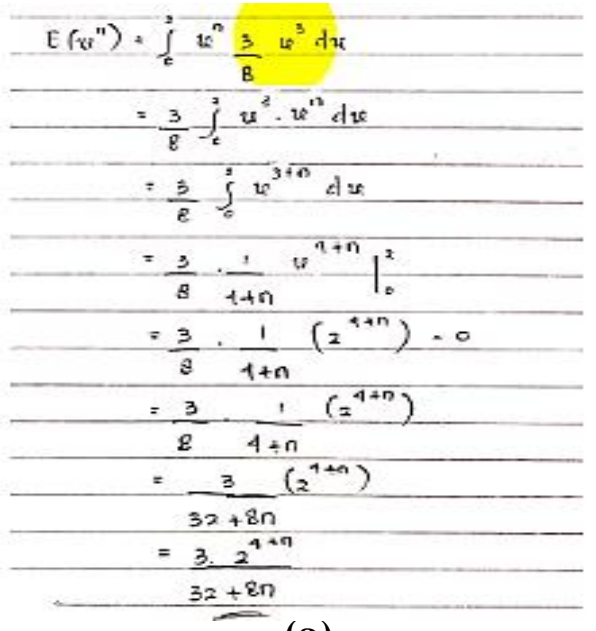

(a)

$\Rightarrow E(x)=\int_{0}^{1} x \frac{3}{8} x^{2} d x$
$\frac{3}{2}=\frac{3}{8} \int_{0}^{2} x^{3} d x$
$\frac{3}{2} \cdot \frac{8}{3}=\int_{0}^{2} x^{3} d x$
$4=\int_{0}^{2} x^{3} d x$
$4=\left.\frac{1}{4} x^{4}\right|_{0} ^{2}$

(b)

Figure 5. $S_{1}$ and $S_{2}$ answers on indicator 4

Based on Figure 5 (a), it can be seen that S1 wrote sequentially the steps to get the general form of $\mathrm{E}\left(\mathrm{X}^{\mathrm{n}}\right)$, but the density function is still wrong. The following are the results of interviews with one of subjects 1 (S1) on indicator 4.

P: "For question number 3, how difficult do you think it is? Easy, moderate, or hard?"

S1: "It is hard, ma'am"

$P$ : "What makes it hard?".

S1: "I have never worked on a question like this, I was confused about how to do this. Usually, the question is looking for the expectation value, but it is not ma'am"

$P$ : "Try to explain again how to get the answer"

S1: "First I look for the value of $E(X)$ and $E\left(X^{2}\right)$ based on the given. Then try to find $E\left(X^{n}\right)$ and use the expectation formula. But I was wrong when determining the general formula Ma'am, it should be $x^{2}$, but it is not, so it is wrong.

Based on the results of the interview, S1 considered that the question number 4 was hard because it was the first time seeing a question like that and was confused how to solve it. When asked to explain the steps for solving the question number 4, S1 can explain to find a general form, the formula for the density function can be used using the concept of integrals, but it was mistaken to write exponents in the density function.

Based on Figure 5 (b), S2 only wrote $\mathrm{E}(\mathrm{X})$ but did not continue the answer until $\mathrm{E}\left(\mathrm{X}^{\mathrm{n}}\right)$. The following are the results of interviews with one of subjects 2 (S2) on indicator 4.

P: "For question number 4, how difficult do you think it is? Easy, moderate, or hard?"

S2: "It is hard, ma'am"

$P$ : "What makes it hard?".

S2: "It is hard because it determines the right formula and determines c"

$P$ : "Try to explain again how to get the answer"

S2: "By following the formula in the textbook page 127 and determining $c$, calculating $E(x)$, find the value of $E\left(x^{2}\right)$, find the $E\left(X^{n}\right)$, but I was confused fiding the $E\left(X^{n}\right)$,

S2 said that the question in number 5 was hard so that he was confused about it, and S2 only chose simple questions as an exercise to complete. While S3 did not answer the question number 5 on the answer sheet. The following are the results of interviews with one of subjects 3 (S3) on indicator 4. 
P: "For question number 4, why did not you answer?"

\section{S3: "I have a hard time using integral ma'am, I am lack of integral material"}

When asked about the integral formula, for example $\int x^{n} d x, \mathrm{~S} 3$ also forgot and took a few minutes to be able to answer. So, based on the results of the analysis of the test results, interview results, and observations of the three subjects, it can be said that the difficulty level on the indicator of "The formation of new concepts related to other concepts" is hard, and only the subjects of S1 who can answer, while S2 and S3 cannot answer.

Based on the previous description, from the triangulation analysis (the results of mathematical abstract thinking abilities test, observations, and interviews) of research subjects who represent each disposition categories, the results show that: 1) S1 is in the high mathematical disposition category. S1 believes that they can solve difficult probability theory questions and feel curious when they cannot solve difficult PTP questions, S1 looks for various references both from sources in handbooks and other sources, and prefers to solve questions related to everyday problems and story questions because they feel more challenged. S1 also asks themselves about the relevance of the answers to the questions asked. As a result, $\mathrm{S} 1$ does not experience significant difficulties in solving mathematical abstract thinking problems. S1 only made a little mistake in answering the question on the indicator of "Construct a general form related to the mathematical process (generalization)". It means that high mathematical disposition causes $\mathrm{S} 1$ to feel eager to continue learning probability theory, so that the ability to think mathematically abstraction tends to be high; 2) S2 is in the medium mathematical disposition category. S2 chooses to work on questions like the existing examples, only chooses simple probability theory questions as an exercise to solve, and is more confident when comparing their own answers with friends' answers. S2 can solve abstract thinking problems well on the indicators of "Transforming problems into symbols" and "Manipulation process of abstract mathematical objects", but has a problem on solving questions on the indicators of "The formation of mathematical concepts related to other concepts" and "Construct a general form related to the mathematical process (generalization)". It means that the medium mathematical disposition is causing S2 still doubtful on their own answer and they will be more confident if they compare their answers with friends, so that their ability to think mathematically abstraction tends to be medium; 3 ) $\mathrm{S} 3$ is in the low disposition category. S3 is hesitant about getting a good score in a probability theory course, and feels hopeless about a difficult PTP problem. S3 can only answer the questions on the indicator of "transforming problems into symbols" correctly. On the indicator of "Manipulation process of abstract mathematical objects" is not quite right, while on the indicators of "The formation of mathematical concepts related to other concepts" and "Construct general forms related to the mathematical process (generalization) "did not answer. It means that a low mathematical disposition causes S3 to be less confident, less diligent and persistent and has less serious concern to the probability theory course, so that the ability to think mathematically abstraction tends to be low.

Then the hardest experienced by students is in indicators of construct a general form related to the mathematical process (generalization). Generalizing a concept means removing a number of attributes from a particular concept. This operation implies an expansion of the conceptual scope and a more general form of the concept (Scheiner, 2016b). Based on this description, it can be said that students consider an important learning topics if they can apply 
them in everyday life or are related to academics and careers and their interests. (Songsore and White, 2018). Therefore it is important to ensure that after students get a basic course in statistics, they will have high interest, positive perceptions and the ability to connect various topics to real-life contexts. (Songsore and White, 2018). Other researches have shown that students who have a realistic view of their abilities tend to perform better while those with less self-confidence are more likely to be poor in maths performance. (Colita and Genuba, 2019).

Then, the hardest experienced by students in solving mathematical abstract thinking problems is on the indicator of "Construct a general form related to the mathematical process (generalization)". Krutetskii states that in order for students to formulate generalizations correctly, they do it to abstract from certain content, and choose equations, structures, and relationships. Another discovery from previous research is that the generalized behavior of students depends on the ability to work in the problem solving for a long time on each problems (Sriraman, 2004). It means that the more often students solve problems related to these problems, the more they will improve their ability to think mathematically abstraction.

\section{CONCLUSIONS}

Based on the analysis results and the discussion, it can be concluded that $\mathrm{S} 1$ is in the high mathematical disposition category, fulfills three indicators of abstract thinking abilities, namely transforming problems into symbols, manipulation process of abstract mathematical objects, the formation of mathematical concepts related to other concepts. S2 is in the medium mathematical disposition category, fulfilling two indicators of abstract thinking abilities, namely transforming problems into symbols and manipulation process of abstract mathematical objects, forming mathematical concepts related to other concepts. S3 is in the low mathematical disposition category, it only fulfills one indicator of the abstract thinking abilities, namely transforming problems into symbols. The hardest experienced by students is in indicators of construct a general form related to the mathematical process (generalization). Teachers and lecturers are expected to be able to explore deeper related to how students actively construct knowledge to form good cognitive structures, through appropriate approaches, models, or strategies so that they can develop their mathematical disposition and abstract thinking abilities.

This research is limited to describing students abstract thinking abilities in terms of mathematical disposition. Recommendations for future research are possible to carry out similar research in different subjects to find new knowledge and add the relationship of student mathematical dispositions with abstract thinking abilities to the literature.

\section{AUTHOR CONTRIBUTIONS STATEMENT}

AN worked as the main drafter in this research. data collection and instrument design assisted by LA and NN.

\section{REFERENCES}

Adelia, V., Susanti, E., Sari, N., \& Simarmata, R. H. (2020). Abstraction ability in number patterns problems. In Journal of Physics: Conference Series (Vol. 1480, No. 1, p. 012049).

Almerino Jr, P. M., Etcuban, J. O., De Jose, C. G., \& Almerino, J. G. F. (2019). Students' affective belief as the component in mathematical disposition. International Electronic Journal of Mathematics Education, 14(3), 475-487. 
Cheng, M. H. M., \& Wan, Z. H. (2017). Exploring the effects of classroom learning environment on critical thinking skills and disposition: A study of Hong Kong 12th graders in Liberal Studies. Thinking Skills and Creativity, 24, 152-163.

Colita, M., \& Genuba, R. L. (2019). School climate and mathematical disposition of grade 10 students. International Journal of Trends in Mathematics Education Research, 2(4), $173-178$.

Darwish, A. H. (2014). The abstract thinking levels of the science-education students in gaza universities. In Asia-Pacific Forum on Science Learning and Teaching 15(2), 1-24.

Debnath, L., \& Basu, K. (2015). A short history of probability theory and its applications. International Journal of Mathematical Education in Science and Technology, 46(1), 1339.

Dietz, H. M. (2016). Metacognitive support of mathematical abstraction processes. In COGNITIVE 2016: The Eighth International Conference on Advanced Cognitive Technologies and Applications Metacognitive, pp. 62-65.

Fitriani, N., \& Nurfauziah, P. (2019). Gender and mathematical abstraction on geometry. In Journal of Physics: Conference Series, 1315(1).

Hakim, L. L., \& Nurlaelah, E. (2018). Mathematical mindsets: The abstraction in mathematical problem solving. In Journal of Physics: Conference Series, 1132(1), 1-5.

Hong, J. Y., \& Kim, M. K. (2016). Mathematical abstraction in the solving of ill-structured problems by elementary school students in Korea. Eurasia Journal of Mathematics, Science and Technology Education, 12(2), 267-281.

Komala, E. (2018). Analysis of students'mathematical abstraction ability by using discursive approach integrated PEER instruction of structure algebra II. Infinity Journal, 7(1), 2534.

Kontou, E., \& Stylianides, N. (2020). Bayes theorem and its recent applications. Leicester Undergraduate Mathematical Journal, 2.

Lestari, S. D., Kartono, K., \& Mulyono, M. (2019). Mathematical literacy ability and mathematical disposition on team assisted individualization learning with RME approach and recitation. Unnes Journal of Mathematics Education Research, 8(2), 157164.

Lin, S. W., \& Tai, W. C. (2016). A longitudinal study for types and changes of students' mathematical disposition. Universal Journal of Educational Research, 4(8), 1903-1911.

Nurhasanah, F., Kusumah, Y. S., Sabandar, J., \& Suryadi, D. (2017). Mathematical abstraction: Constructing concept of parallel coordinates. In Journal of Physics: Conference Series, 895(1).

Nurhasanah, F., Kusumah, Y. S., Sabandar, J., \& Suryadi, D. (2018). Relationship between mathematical abstraction in learning parallel coordinates concept and performance in learning analytic geometry of pre-service mathematics teachers: An investigation. In Journal of Physics: Conference Series, 1013(1). 
Putra, J. D., Suryadi, D., \& Juandi, D. (2018). Mathematical abstraction ability of prospective math teacher students. In Journal of Physics: Conference Series, 1132(1).

Sa'diyah, M., Sa'dijah, C., \& Handayani, U. (2019). How students build their mathematical dispositions towards solving contextual and abstract mathematics problems. In Journal of Physics: Conference Series, 1397(1).

Salgado, H., \& Trigueros, M. (2015). Teaching eigenvalues and eigenvectors using models and APOS Theory. The Journal of Mathematical Behavior, 39, 100-120.

Salido, A., \& Dasari, D. (2019). The analysis of students' reflective thinking ability viewed by students' mathematical ability at senior high school. In Journal of Physics: conference series, 1157(2).

Scheiner, T. (2016a). New light on old horizon: Constructing mathematical concepts, underlying abstraction processes, and sense making strategies. Educational Studies in Mathematics, 91(2), 165-183.

Scheiner, T. (2016b). New light on old horizon: Constructing mathematical concepts, underlying abstraction processes, and sense making strategies. Educational Studies in Mathematics, 91(2), 165-183.

Scheiner, T., \& Pinto, M. M. (2016). Images of abstraction in mathematics education: contradictions, controversies, and convergences. In 40th Conference of the International Group for Psychology of Mathematics Education (pp. 155-162). International Group for the Psychology of Mathematics.

Songsore, E., \& White, B. J. (2018). Students'perceptions of the future relevance of statistics after completing an online introductory statistics course. Statistics Education Research Journal, 17(2), 120-140.

Sriraman, B. (2004). Reflective abstraction, uniframes and the formulation of generalizations. The Journal of Mathematical Behavior, 23(2), 205-222.

Wang, F., \& XU, X. (2018). Research on the teaching of probability theory and mathematical statistics for non-statistics majors. In 2018 International Conference on Education Reform and Management Science (ERMS 2018). 177(Erms), pp. 208-212.

Widada, W., Agustina, A., Serlis, S., Dinata, B. M., \& Hasari, S. T. (2019). The abstraction ability of students in understanding the concept of geometry. In Journal of Physics: Conference Series, 1318(1).

Yaniawati, R. P., Indrawan, R., \& Setiawan, G. (2019). Core model on improving mathematical communication and connection, analysis of students' mathematical disposition. International Journal of Instruction, 12(4), 639-654.

Yilmaz, R., \& Argun, Z. (2018). Role of visualization in mathematical abstraction: The case of congruence concept. International Journal of Education in Mathematics, Science and Technology, 6(1), 41-57. 